\title{
Multiple Iterations of Bundle Adjustment for the Position Measurement of Fiber Tips on LAMOST
}

\author{
Mingchi Feng ${ }^{1}$, Yonggang $\mathrm{Gu}^{2}$, Yi Jin², Chao Zhai ${ }^{2}$ \\ ${ }^{1}$ Department of Precision Machinery and Precision Instrumentation, University of Science and Technology of China, \\ 230027, Hefei, China, fmc105@mail.ustc.edu.cn \\ ${ }^{2}$ Experimental Center of Engineering and Material Sciences, University of Science and Technology of China, 230027, \\ Hefei, China, yggu@ustc.edu.cn (corresponding author), yjin@ustc.edu.cn, zhaichao@ustc.edu.cn
}

\begin{abstract}
In the astronomical observation process of multi-object fiber spectroscopic telescope, the position measurement of fiber tips on the focal plane is difficult and critical, and is directly related to subsequent observation and ultimate data quality. The fibers should precisely align with the celestial target. Hence, the precise coordinates of the fiber tips are obligatory for tracking the celestial target. The accurate movement trajectories of the fiber tips on the focal surface of the telescope are the critical problem for the control of the fiber positioning mechanism. According to the special structure of the LAMOST telescope and the composition of the initial position error, this paper aims at developing a high precision and robust measurement method based on multiple iterations of bundle adjustment with a few control points. The measurement theory of the proposed methodology has been analyzed, and the measurement accuracy has been evaluated. The experimental results indicate that the new method is more accurate and more reliable than the polynomial fitting method. The maximum position error of the novel measurement algorithm of fiber tips with simulated and real data is $65.3 \mu \mathrm{m}$, and most of the position errors conform to the accuracy requirement $(40 \mu \mathrm{m})$.
\end{abstract}

Keywords: Bundle adjustment, LAMOST, multiple iterations, position error, position measurement.

\section{INTRODUCTION}

$\mathrm{W}$ ITH THE CONTINUOUS DEVELOPMENT of the multi-object fiber spectroscopic telescope, the integrated number of optical fibers on the focal plane is increasing, from 400 of AAT [1] to 640 of SDSS [2], 4000 of LAMOST [3], and 5000 of BIGBOSS [4]. As an important part of these telescopes, the fiber positioning system is capable of actuating the fiber-positioner and targeting the celestial object. With the consecutive increase of the fiber number and the operation speed, the fiber positioning cannot be accomplished artificially anymore. Therefore, the LAMOST has developed the parallel controllable fiber positioning technique. Aiming to control the fiber tips on the focal surface of the telescope accurately, the movement trajectories of the fiber tips must be accurately measured in advance, so the crucial problem is the fiber position measurement. This paper introduces a measurement method with high precision and robustness by using the bundle adjustment (BA) method with a few control points.

At present, the position measurement methods include the contact measurement and the non-contact measurement. The contact measurement with a high measuring precision has been widely adopted, such as the laser tracker and the coordinate measuring machine (CMM). C. Read [5] utilized four laser tracker measurement systems for the surface-panel segments and developed a new method of analysis which was designated as the Mahalanobis Bundle Adjustment [6]. R. D'Amato [7] used CMM to measure angles by the geometric characterization of perpendicular planes and estimated the uncertainty of angle measurements. There are numerous fiber-positioners on the focal plane with micron diameter fibers, therefore, the efficiency and applicability of the traditional contact measurement method such as CMM and the laser tracker are insufficient to measure the fiber tip position.

The vision measurement method featured with high precision and high efficiency can overcome these difficulties. This method can be classified as the linear CCD (Charge Coupled Device) measurement, the single view measurement, and the multi-view measurement. Shi et al. [8] proposed a high-speed measurement algorithm based on the linear CCD for the position of holes on a large plane. The high-resolution linear CCD associated with a grating ruler scanned the plane to be measured and grabbed the overall image of the holes on the plane. The fiberpositioners are distributed on the focal plane of 1.75 meters in diameter and the focal plane has no space to install the linear CCD. A lot of stress and strain measurement methods are based on the single view [9], [10]. In the past years, several kinds of vision measurement methods for the LAMOST's fiber position based on the single camera have been presented [11], [12], and these methods are based on the polynomial fitting method. The multi-view measurement method has been widely used. The subpixel edge detections of coded points properly distributed on complex surfaces are applied in the common marker-based method. This methodology is suitable for various applications, though it is typically based on time-consuming procedures [13], [14]. Recently, a developed system merged stereo vision and fringe projection to measure a large area free form surfaces with a freely definable density of points expressed in a global coordinate system [13]. This system relied on highprecision Digital Light Procession (DLP) projector and the measuring precision cannot meet the requirement for fiber positioning. Moreover, the stereo vision and the fringe projection cannot measure complex surfaces like the LAMOST focal plane (as shown in Fig.1.). 
Because all these methods are passive methods and the diameter of the fiber is micron scale, the direct feature point extraction will bring more errors into the measuring result. Fortunately, the fiber position measurement with the help of backward lighting is feasible, in which the fibers transfer the backward lighting to the measured fiber tips, and then, the camera takes pictures of the telescope focal plane. So this paper develops an active multi-view measurement method based on the bundle adjustment to improve the precision of the fiber position measurement.

In general, sensor models are classified into two categories: the physical sensor model and the alternative generalized model [15]. The physical camera model, based on the collinearity condition, describes the rigorous imaging geometric relationship between the image point and the object point with parameters of physical meanings. In contrast, the polynomial fitting method belongs to the alternative generalized models utilizing only one view. The polynomial fitting method supplied with calibrated polynomial coefficients, expresses the object point coordinates as a ratio of two polynomials with variables of image coordinates. In practice, the polynomial fitting method is widely applied due to its capability of maintaining the accuracy of the physical sensor models and real-time calculation. But the coefficients of the polynomial fitting method must be obtained based on the known high-precision 3D (3 Dimensions) points on the focal plane, and the image plane must keep parallel to the measuring surfaces. This method is only suitable for the measurement of planar object. Inconvenient actual use and ambiguous physical meaning of the parameters will affect the accuracy of the measurement results. In this paper, the accuracy of the proposed method and the polynomial fitting method will be compared in the same situation.

In order to improve the measurement accuracy and adaptability, this paper develops a new method based on the multi-view reconstruction and the bundle adjustment to measure the $3 \mathrm{D}$ coordinates of fiber tips, in which the bundle adjustment is the most critical step. The bundle adjustment is the choice for many photogrammetry applications [16]-[18]. It has also come to take a prominent role in computer vision applications as the last step of many feature-based 3D reconstruction algorithms [19]-[21]. BA refines a visual reconstruction to produce jointly optimal $3 \mathrm{D}$ structure and viewing parameter (camera pose and intrinsic camera parameters) estimates. BA boils down to minimizing the re-projection error between the observed and predicted image points, which is expressed as the sum of squares of all the residual feature projection errors [16]. Thus, the minimization is achieved by using non-linear least squares algorithms, of which most of these articles apply the Levenberg-Marquardt (LM) algorithm [22].

Based on the special structure (as shown in Fig.1.) of the LAMOST telescope and the composition of the initial position error, this paper analyzes a novel bundle adjustment model with additional constraints of abundant measuring points and only one high-precision control point. The method of reducing the systematic error and the zero-mean Gaussian random error is presented, and the theoretical and actual experiment accuracy of this method is assessed. This paper is organized as follows: The mathematical models of camera and bundle adjustment are presented in Section 2. This section also describes the composition of the initial position error and comes up with the methods to reduce the error. Section 3 provides experimental results with both simulated and real data. Finally, Section 4 presents the conclusions.

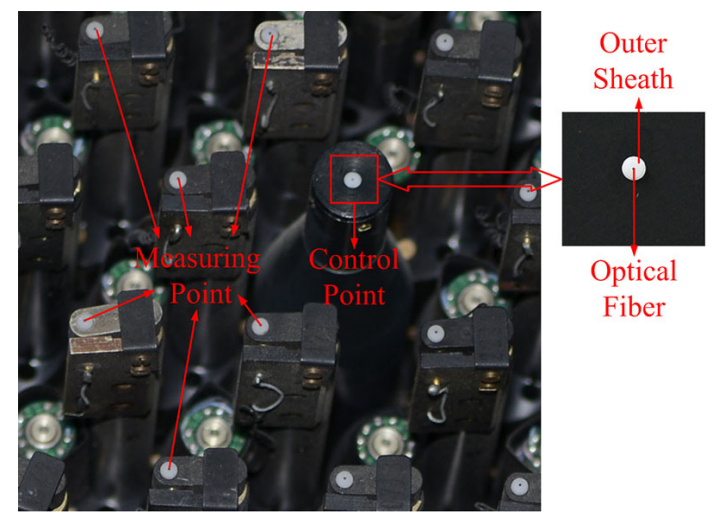

Fig.1. The special structure of the LAMOST focal plane.

\section{MEASUREMENT PRINCIPLE \& MATHEMATICAL MODELING}

The camera model is the core part to the new method. The camera should be accurately calibrated to obtain consistent intrinsic and relative orientation parameters. A network of images is taken from successive locations as the camera is moved around an object. Corresponding image feature points extracted from these images and the initial estimate of 3D object coordinates are used to measure the position of fiber tips, which are inserted into bundle adjustment with control points being geometric constraints. We will now briefly discuss the measurement process over multiple views and the proposed method. This section firstly introduces some basic theories, including camera calibration, bundle adjustment, initial estimates, and the iteration theory with control points. The last part of this section introduces the polynomial fitting method currently employed in the measurement.

\section{A. Camera model and camera calibration.}

The common mathematical model of camera and the notation used in this paper are presented firstly. A camera is modeled by the pinhole: the relationship between a $3 \mathrm{D}$ point $[X, Y, Z]$ and its image projection $[u, v]$ is approximated by means of the rotation matrix and transformation matrix, as shown in (1):

$$
\begin{gathered}
{\left[\begin{array}{lll}
X_{c} & Y_{c} & Z_{c}
\end{array}\right]^{T}=R *\left[\begin{array}{lll}
X & Y & Z
\end{array}\right]^{T}+T} \\
Z_{c}\left[\begin{array}{lll}
u & v & 1
\end{array}\right]^{T}=A\left[\begin{array}{lll}
X_{c} & Y_{c} & Z_{c}
\end{array}\right]^{T} \\
=\left[\begin{array}{ccc}
f_{u} & \lambda & u_{0} \\
0 & f_{v} & v_{0} \\
0 & 0 & 1
\end{array}\right]\left[\begin{array}{lll}
X_{c} & Y_{c} & Z_{c}
\end{array}\right]^{T}
\end{gathered}
$$


Where, $(X, Y, Z)$ are the object world coordinates of a measured point and $(u, v)$ are its pixel image coordinates, and $\left(X_{c}, Y_{c}, Z_{c}\right)$ are its camera coordinates. $Z_{c}$ is a nonzero scale factor. $\left(u_{0}, v_{0}\right)$ denote the principal point in the imaging plane with the unit of pixel. $f_{u}$ and $f_{v}$ represent the focal length in pixels along the image axes $u$ and $v$, while $\lambda$ is the skew coefficient defining the angle between the $u$ and $v$ pixel axes. $A$ is the intrinsic camera parameter. $R$ and $T$, called the extrinsic parameters, are the rotation matrix and the translation vector from world coordinate frame to camera coordinate frame, respectively. The initial estimate of camera parameters can be calculated by the direct linear transformation (DLT) method directly.

Actually, the real camera imaging is not ideal, particularly when a commercial Single Lens Reflex (SLR) camera is used. Therefore, the lens distortion on the imaging has to been taken into account. Commonly, only first-order or second-order distortion model is adopted to correct the radial distortion [23]-[25]. More rigorously, the radial distortion and tangential distortion should be adopted to correct the lens distortion [26], [27]. After considering the lens distortion, the new normalized point coordinates $\left(x_{d}, y_{d}\right)$ are defined as follows.

Let $(x, y)^{\mathrm{T}}$ and $\left(x_{d}, y_{d}\right)^{\mathrm{T}}$ be the distortion-free and the distorted normalized image coordinates, respectively.

$$
\begin{gathered}
{\left[\begin{array}{l}
x \\
y
\end{array}\right]=\left[\begin{array}{l}
X_{c} / Z_{c} \\
Y_{c} / Z_{c}
\end{array}\right]} \\
{\left[\begin{array}{l}
x_{d} \\
y_{d}
\end{array}\right]=\left(1+k_{1} r^{2}+k_{2} r^{4}+k_{5} r^{6}\right)\left[\begin{array}{ll}
x & y
\end{array}\right]^{T}+d x} \\
d x=\left[\begin{array}{l}
2 k_{3} x y+k_{4}\left(r^{2}+2 x^{2}\right) \\
k_{3}\left(r^{2}+2 y^{2}\right)+2 k_{4} x y
\end{array}\right]
\end{gathered}
$$

where, $r^{2}=x^{2}+y^{2} .1+k_{1} r^{2}+k_{2} r^{4}+k_{5} r^{6}$ is radial distortion coefficient and $d x$ is the tangential distortion.

$$
\left\{\begin{array}{c}
u_{d}=f_{u}\left(x_{d}+\lambda * y_{d}\right)+u_{0} \\
v_{d}=f_{v} y_{d}+v_{0}
\end{array}\right.
$$

$k_{1}, k_{2}, k_{5}$ are the coefficients of radial distortion, and $k_{3}, k_{4}$ are the coefficients of tangential distortion. $\left(u_{d}, v_{d}\right)$ are the distorted pixel image coordinates. It requires an initial guess of intrinsic and extrinsic camera parameters, which can be obtained using the DLT method described by Hartley [28]. Then, the best estimate for the camera parameters can be obtained by nonlinear refinement based on the maximum likelihood criterion, such as the LM algorithm. In the simulation of the computational methods, inter-image homographies are computed with normalized DLT from perturbed image points. The initial value of the intrinsic parameters has also been determined with the assumption that the images are without distortion [29].

\section{B. Bundle adjustment}

A new bundle adjustment problem with control point is described in this paper. The direct application of a traditional bundle adjustment which is influenced by various errors fails to get the accurate 3D coordinates. However, if the control point constraints are applied to the traditional bundle adjustment, BA will get better camera parameters and $3 \mathrm{D}$ point coordinates. BA utilizing the control point constraints can reduce the matrix dimension of the covariance matrix so that the total computation time is decreased. Experiment results indicate that the new method is better than the traditional bundle adjustment algorithm in the $3 \mathrm{D}$ accuracy and the convergence rate.

Let $f$ be an assumed functional relation of the camera, and we can get the reprojection error of an image feature point $e_{i j}=x_{i j}-f\left(R, T, K, X_{i j}\right)$, which is used to evaluate $3 \mathrm{D}$ reconstruction quality. $x_{i j}$ is the actual image point coordinate of point $i$ on image $j ; R, T$ are the extrinsic camera parameters; $K$ presents the intrinsic camera parameters and distortion coefficients in (2), (4); $X_{i j}$ is the initial estimate of a fiber tip's position; and $f\left(R, T, K, X_{i j}\right)$ is the predicted image coordinate according to (6).

Further refinement can be conducted with bundle adjustment. It can be defined as the problem of simultaneously refining the 3D structure and viewing parameters (i.e., camera pose, intrinsic parameters, and distortion) of the cameras employed to acquire the images. And, BA in general is based on a non-linear iterative minimization algorithm (e.g., Levenberg-Marquardt method) and is used as the final step of the reconstruction process. In order to get precise results, it requires an initial solution that can arise from the steps described above. Moreover, it is possible to provide the algorithm with the covariance of each measuring point so as to minimize the Mahalanobis distance of the reprojection error [30] :

$$
E=\sum_{i}^{n} \sum_{j}^{m}\left\|x_{i j}-f\left(R, T, K, X_{i j}\right)\right\|^{2}
$$

Where, it is assumed that $n$ 3D points are seen in $m$ views. In this paper, the nonlinear minimization problem is solved with the LM algorithm [22].

\section{Estimating initial camera parameters and $3 D$ object coordinates.}

Initial estimates include the $3 \mathrm{D}$ coordinates of the object point, intrinsic and extrinsic camera parameters, and camera lens distortion. Better initial values of the bundle adjustment can avoid the solution falling into local optimization. The improvement of the initial solution accuracy of the camera parameters and the coordinates of $3 \mathrm{D}$ points is an essential step in the bundle adjustment optimization, which calls for the application of nonlinear optimization after the DLT method in camera calibration.

The camera intrinsic parameters and lens distortion can be obtained by the aforementioned camera calibration method, and the designed 3D coordinates of the measuring points can be used as the initial coordinates. In actual measurement, 
extrinsic camera parameters are calculated by the DLT method with the designed 3D coordinates of the measuring points and the corresponding image coordinates. And the relatively accurate extrinsic camera parameters can also be determined by some control points in the field of view. The initial position of a specific fiber tip can be easily obtained by its corresponding revolving angles of the central revolving axle and the bias revolving axle in the double parallel controllable structure. The initial fiber positions of the test plane are the designed 3D coordinates.

\section{Bundle adjustment with control points and its iterative process.}

With the absence of additional constraints, the traditional bundle adjustment cannot meet the precision request. All the 3D points are divided into control points and measuring points, while most of them are measuring points and only a few are control points (as shown in Fig.1.). Half of the measuring points are treated as a large number of low precision control points. Assuming that the difference between the initial coordinates and the actual coordinates of $3 \mathrm{D}$ points is non-zero mean Gaussian, the difference of half points is also non-zero mean Gaussian. The calculation process is as follows: (1) First, half of all points are used as low precision control points, and the other half are measuring points, then the position errors are reduced and the average of these errors would converge to the mean value of the control point errors after carrying out the bundle adjustment. (2) Then, the roles of control points and measuring points are reversed and the position of $3 \mathrm{D}$ points is iteratively calculated and analyzed by bundle adjustment. When the error is no longer reduced, the process stops and it can be regarded as the coarse solution. (3) Finally, if there is any position error that cannot meet the requirement, this point acts as a measuring point and the other points act as control points, then BA will be carried out. This step can be regarded as the finishing solution.
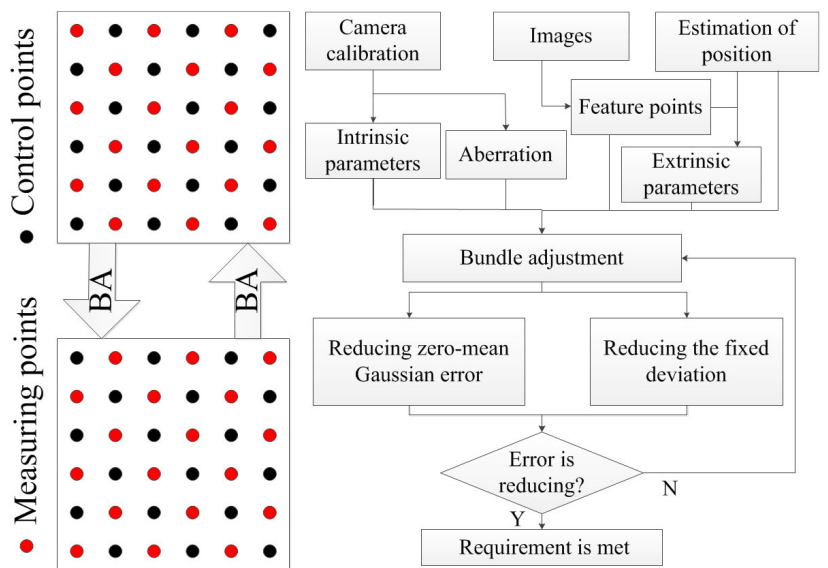

Fig.2. The flowchart of the measurement process.

\section{E. The influence of the systematic error and its elimination method.}

The actual initial position error of $3 \mathrm{D}$ points is non-zero mean Gaussian. The position error consists of the systematic error and the zero-mean Gaussian random error. The above method can greatly reduce the zero-mean Gaussian random error and the error of measuring points converges to the mean of the position errors of control points. But this method is incapable of reducing the systematic error and the systematic error will be brought into the final results. In order to eliminate the systematic error, a high-precision control point measured by high-precision CMM is set up in the measurement area. The BA results and the accurately measured results of this point are applied to calculate the systematic error, which is used for compensation of all measuring points so as to improve the accuracy of the results. The flowchart of the measurement process is shown in Fig.2.

The actual coordinates of $3 \mathrm{D}$ points are simulated by adding the zero-mean Gaussian random error and the systematic error into the theoretical coordinates. The calculation results show that the systematic error is brought into the BA results. The control point coordinates are determined in advance by CMM. The BA results and the previous measured coordinates of the control point are used to get the systematic error which is available for compensation of the other points coordinate. A large number of measuring points with zero-mean Gaussian random error determine the scale and direction of all points, while the high-precision control point determines the systematic error of all points.

\section{F. Fourth-order polynomial model.}

Due to the advantages of real-time calculation and high precision, the polynomial fitting method is employed in the LAMOST project to measure the coordinates of fiber tips, but it also has the disadvantage of weak adaptability. As a comparison object, this section simply introduces the realization of the polynomial fitting method. The fourthorder polynomial model is defined as (8). $X$ and $Y$ are the center coordinates of the fiber tip on the focal plane while $u$ and $v$ are the center coordinates of the fiber tip on the image. The least square method is employed to calculate the coefficients $a_{1}$ to $a_{15}$ and $b_{1}$ to $b_{15}$ with at least 15 highprecision known 3D points. Then, we can convert the image coordinate (unit: pixel) to the focal plane coordinate (unit: millimeter) based on the 30 coefficients. The least square criterion is adopted to solve the linear equation. By this method, less computing time is needed and real-time calculation can be carried out. Therefore, the measuring region of the focal plane is with enough accuracy.

$$
\left\{\begin{array}{c}
X=a_{1}+a_{2} u+a_{3} v+\cdots+a_{14} u v^{3}+a_{15} v^{4} \\
Y=b_{1}+b_{2} u+b_{3} v+\cdots+b_{14} u v^{3}+b_{15} v^{4}
\end{array}\right.
$$

\section{EXPERIMENT}

\section{A. Experiment setup and camera calibration.}

Based on the measurement method and analysis, the vision measurement system is set up, as shown in Fig. 3, which consists of a CMOS camera (NIKON D800) with the resolution of 7360 pixels $\times 4912$ pixels, a test board (small focal plane), a calibration board and a translation stage. The $22 \times 22$ circles array is distributed uniformly on the surface of 
calibration board. The distance between adjacent circles is $3 \mathrm{~mm}$. The positioning accuracy of the calibration points is $2.5 \mu \mathrm{m}$. The shooting distance is set to $3 \mathrm{~m}$, and the calibration volume is $500 \mathrm{~mm} \times 500 \mathrm{~mm} \times 500 \mathrm{~mm}$. The angle between the camera and the calibration board is random. Then the calibration board is located and translated by the translation stage, and thirty-one images of the calibration board under different orientations are captured by the camera. The center of the circles is determined by a subpixel edge extraction and ellipse fitting method. With the images of the circles array, the camera is calibrated with the DLT and nonlinear optimization method. Subsequently, another thirty images are taken and different errors are added to the theoretical coordinates of the calibration board to simulate the initial value of the actual situation. Finally, the camera parameters and the coordinates of $3 \mathrm{D}$ points are jointly optimized with bundle adjustment which optimizes the camera parameters while adjusting the coordinates of $3 \mathrm{D}$ points in a global optimization.

The LAMOST pays more attention to the positioning accuracy of planar XY direction on the focal plane. The positioning accuracy of axial direction on the focal plane has no influence on observation, so experiments will only validate the positioning accuracy of $X Y$ direction. The robustness and accuracy of the algorithm presented throughout this paper are checked based on the experiment results. Results of the simulated data are firstly performed. Then, we present some results from real data experiments using a commercial camera. The experiments of real data apply the small focal plane which is shown in Fig.3.

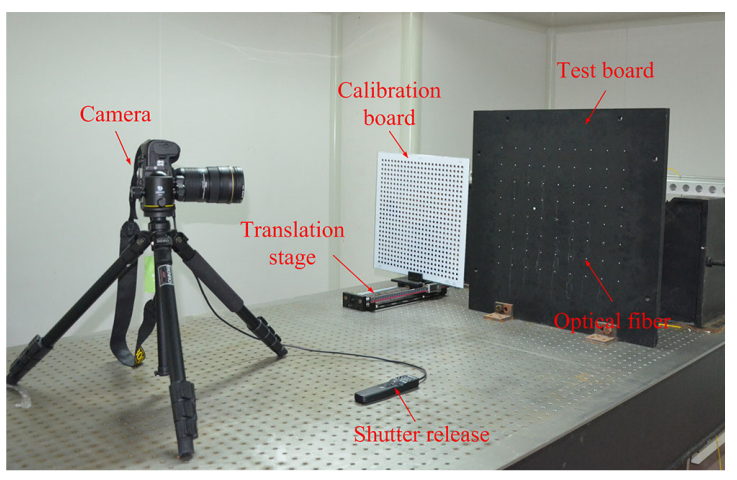

Fig.3. The vision measurement system.

\section{B. Simulated data.}

In order to verify the reliability and accuracy of the new algorithm, the initial position errors of all measuring points are Gaussian distribution with the mean being $500 \mu \mathrm{m}$ and the variance being $200 \mu \mathrm{m}$, as shown in Fig.4.a. The representative pinhole camera model is adopted in this paper, but the actual camera does not strictly comply with this model. If the traditional bundle adjustment without dimension constraints is applied to calculate the coordinates of the measuring points, it is impossible to meet the accuracy requirement. For the purpose of improving the accuracy of position measurement, the dimension constraints, such as predetermined fiber control points, scale and points measured by the laser tracker [31], can be preset. When four accurate control points are employed, it can be observed from Fig.4.b. that the position errors of calculation results are very small, but another thing to note is that the accurate control points are difficult to obtain in practice. When the temperature is constant of $\left(25.5 \sim 26.5^{\circ} \mathrm{C}\right)$, the eight fixed optical fibers (its structure is shown in Fig.1.) are measured by the CMM as control points. The measurement error of CMM is $3 \mu \mathrm{m}$ and the expanded uncertainty is $5.9 \mu \mathrm{m}$ with a confidence level of approximately $95 \%$. When there are not enough control points for constraints, the traditional bundle adjustment is incapable to effectively improve the measurement precision. However, a mass of control points would reduce the number of observation fibers and influence the results of astronomical observation. The bundle adjustment with a mass of low precision control points (measuring points) can reduce zero-mean Gaussian random error but has no effect on the systematic error (as shown in Fig.4.c), while the proposed algorithm with multiple iterations can reduce the zero-mean Gaussian random error and a control point can eliminate the systematic error at the same time. Because each region needs only one high precision control point, it effectively reduces the number of the control points. The proposed algorithm not only improves the measurement precision, but also reduces the influence of control points on astronomical observation. The result is shown in Fig.4.d. The maximum position error is $65.3 \mu \mathrm{m}$, and most of the position errors conform to the accuracy requirement $(40 \mu \mathrm{m})$.

The current measurement method of fiber position in LAMOST is the polynomial fitting method, which requires the image plane being roughly parallel to the object plane. The 3D coordinates of known points and the image feature points are applied to calculate polynomial coefficients based on (8). Then, the XY coordinates of measuring points will be determined by the coordinates of the image feature points and the polynomial coefficients. The polynomial fitting method is suitable for measurement of planar XY coordinates and impracticable to work on $\mathrm{Z}$ direction. In the practical measurement, optical fiber unit has a small displacement $(-0.7 \sim 0.7 \mathrm{~mm})$ in $\mathrm{Z}$ direction. The calibration board is employed to simulate the actual situation. The polynomial coefficients are firstly solved by the calibration board, then the calibration board is moved back $0.5 \mathrm{~mm}$ and the XY coordinates of the corresponding 3D point are calculated based on the polynomial coefficients. The result is shown in Fig.5. The comparison between the proposed bundle adjustment and the polynomial fitting results shows that the bundle adjustment with a mass of low precision control points (measuring points) is more reliable and more accurate. The linear computation and high computation speed are the chief advantages of the polynomial fitting method.

From the measurement results of simulated data listed in Table 1., the experimental results show that the new method and the polynomial fitting method converge reliably. The results with the new method are more accurate than the results with the polynomial fitting method. 


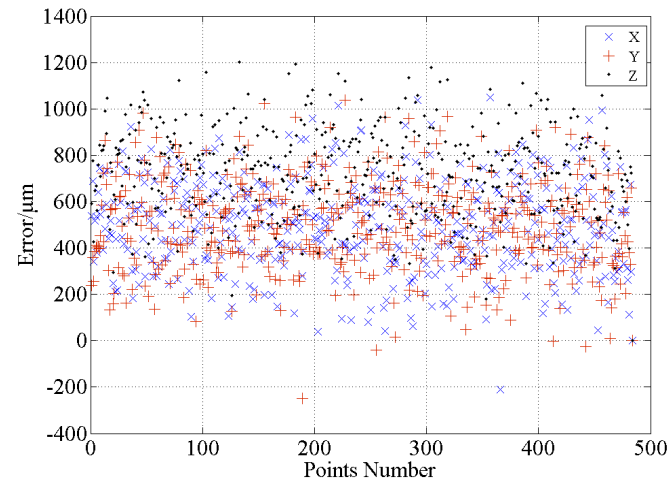

(a) Initial position error.

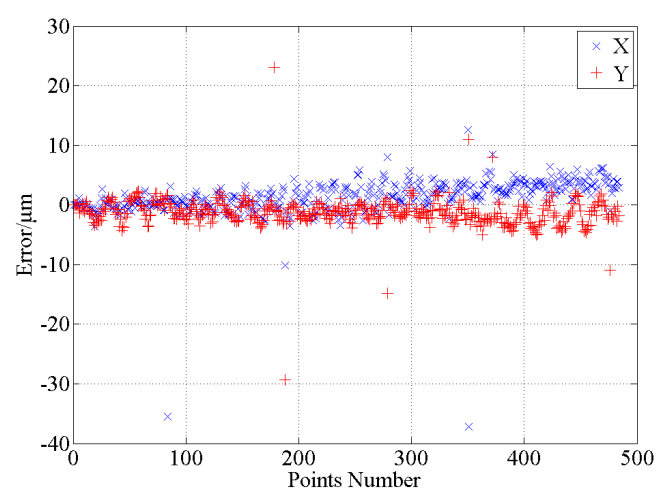

(b) Four high-precision control points.

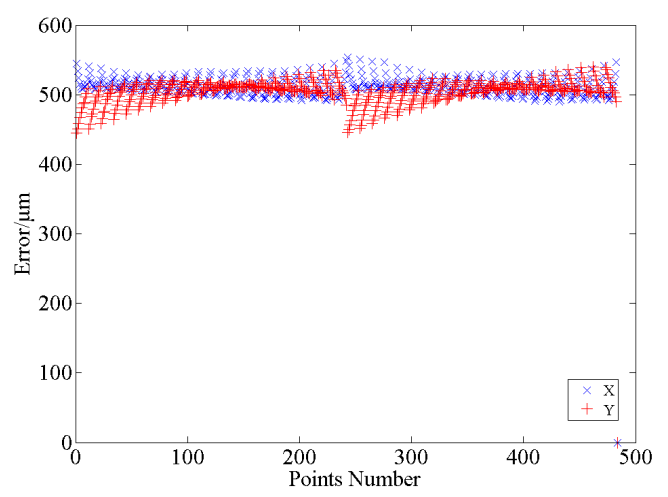

(c) Results before reducing systematic error.

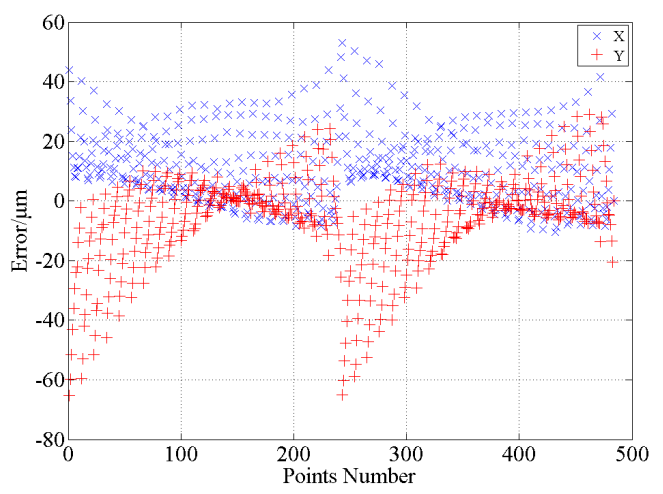

(d) Results after reducing systematic error.

Fig.4. Results of the new bundle adjustment algorithm with simulated data.

\section{Real data.}

The test board processed by high-precision numerical control machine is applied in the actual experiment. The area of this steel plate is roughly equal to the actual measurement area. A series of holes for installing optical fibers are distributed on the steel plate, as shown in Fig.3.

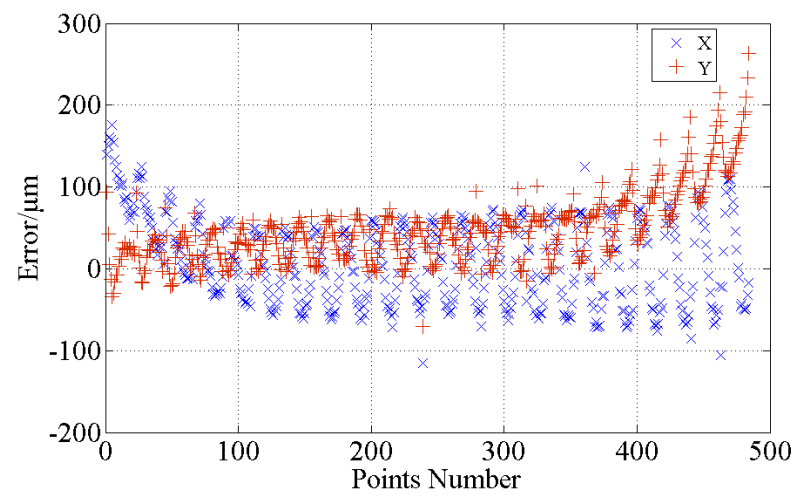

Fig.5. Results of the polynomial fitting method with simulated data.

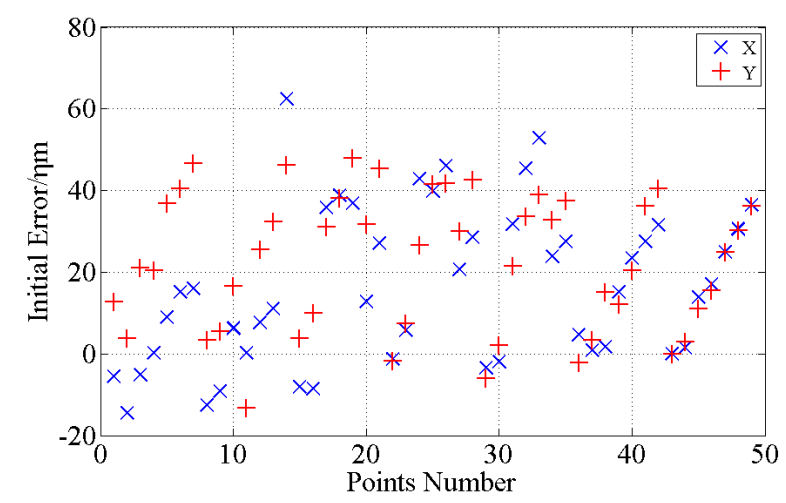

(a) Initial position error.

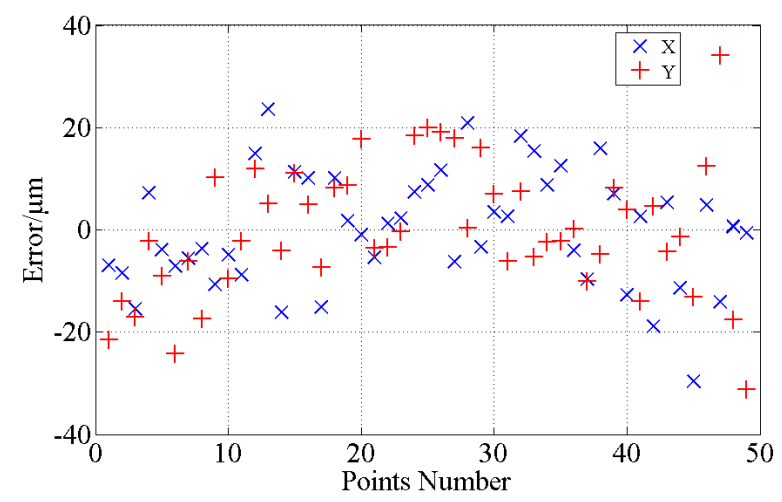

(b) Results with real data.

Fig.6. Result of the new bundle adjustment algorithm with real data. 
The positions of holes have been measured by highprecision CMM. The outer sheath of fiber tip is closely installed in the hole, so the position of the hole is the position of the fiber. By comparison with the results of the present algorithm (as shown in Fig.6.) and CMM, it shows that the maximum position error is $34.1 \mu \mathrm{m}$, and all position errors conform to the accuracy requirement $(40 \mu \mathrm{m})$. Table 2 . shows that the new method converges reliably with real data. But this result is less obvious than the simulated data experiment. It is because the initial position errors of measuring points are relatively small.

Table 1. Measurement results of simulated data (unit: $\mu \mathrm{m}$ ).

\begin{tabular}{|c|c|c|c|c|}
\hline \multicolumn{2}{|c|}{} & $\begin{array}{c}\text { Initial } \\
\text { error }\end{array}$ & $\begin{array}{c}\text { BA } \\
\text { results }\end{array}$ & $\begin{array}{c}\text { polynomial } \\
\text { fitting }\end{array}$ \\
\hline $\begin{array}{c}\text { Maximum } \\
\text { Abs value }\end{array}$ & $\mathrm{X}$ & 1050.3 & 53.1 & 176.1 \\
\cline { 2 - 5 } & $\mathrm{Y}$ & 1038.3 & 65.3 & 263.2 \\
\hline $\begin{array}{c}\text { Mean } \\
\text { value }\end{array}$ & $\mathrm{X}$ & 494.3 & 11.6 & 9.7 \\
\cline { 2 - 5 } & $\mathrm{Y}$ & 495.6 & -6.2 & 48.0 \\
\hline $\begin{array}{c}\text { Standard } \\
\text { deviation }\end{array}$ & $\mathrm{X}$ & 199.6 & 12.3 & 53.0 \\
\cline { 2 - 5 } & $\mathrm{Y}$ & 198.2 & 15.6 & 44.7 \\
\hline
\end{tabular}

Table 2. Measurement results of real data (unit: $\mu \mathrm{m}$ ).

\begin{tabular}{|c|c|c|c|}
\hline \multicolumn{2}{|c|}{} & $\begin{array}{c}\text { Initial } \\
\text { error }\end{array}$ & $\begin{array}{c}\text { BA } \\
\text { results }\end{array}$ \\
\hline $\begin{array}{c}\text { Maximum } \\
\text { Abs value }\end{array}$ & $\mathrm{X}$ & 62.4 & 29.7 \\
\cline { 2 - 4 } $\begin{array}{c}\text { Mean } \\
\text { value }\end{array}$ & $\mathrm{Y}$ & 48.0 & 34.1 \\
\cline { 2 - 4 } & $\mathrm{Y}$ & 22.5 & -0.1 \\
\hline $\begin{array}{c}\text { Standard } \\
\text { deviation }\end{array}$ & $\mathrm{X}$ & 18.9 & 11.3 \\
\cline { 2 - 4 } & $\mathrm{Y}$ & 16.6 & 13.0 \\
\hline
\end{tabular}

\section{CONCLUSIONS}

The actual initial position errors of 3D points are composed of the systematic error and the zero-mean Gaussian random error relative to the theoretical coordinates. This paper introduces an accurate and robust position measurement method for the fiber tips based on iterative bundle adjustment and additional constraints which includes a large number of low-precision control points (measuring points) and only one high-precision control point. The mathematical models of camera and bundle adjustment are established with introduction of various lens distortion correction models. Then the DLT method is used to obtain the initial estimate of the camera. And, the most rigorous bundle adjustment method is applied to calibrate the camera, while the camera parameters are revised simultaneously to estimate the measuring points more accurately and reliably. The polynomial fitting method is also introduced as a reference method.

Experiments with both simulated and real data indicate that the new method can converge reliably and the new method can provide more accurate coordinates of the measuring points than the polynomial fitting method. Compared with the measurement result of CMM or theoretical value, the maximum position error of the novel measurement algorithm of fiber tips with simulated data is $65.3 \mu \mathrm{m}$, and most of the position errors conform to the accuracy requirement $(40 \mu \mathrm{m})$. The maximum position error with real data is $34.1 \mu \mathrm{m}$, and all the position errors conform to the accuracy requirement. The new method is more accurate and more reliable than the polynomial fitting method in the same situation, and the measuring precision can meet the requirement for fiber positioning. Compared with the polynomial fitting method, the new method has additional advantages. First, less high precision control points are required; second, this method can measure all kinds of surfaces; and third, the changes of temperature and distance have little influence on the measurement accuracy.

\section{REFERENCES}

[1] Gillingham, P.R. (1989). Lessons for new large telescopes from the AAT. Astrophysics and Space Science, 160 (1), 281-286.

[2] Kent, S.M. (1994). Sloan digital sky survey. Astrophysics and Space Science, 217 (1), 27-30.

[3] Xing, X.Z., Zhai, C., Du, H.S., Li, W.M., Hu, H.Z., Wang, R.F., Shi, D.X. (1998). Parallel controllable optical fiber positioning system for LAMOST. In Advanced Technology Optical / IR Telescopes VI, Proc. SPIE 3352, 839-849.

[4] Edelstein, J., Poppett, C., Sirk, M., Besuner, R., Lafever, R., Allington-Smith, J.R., Murray, G.J. (2012). Optical fiber systems for the BigBOSS instrument. In Modern Technologies in Space-and Ground-Based Telescopes and Instrumentation II, Proc. SPIE 8450.

[5] Predmore, C.R. (2010). Bundle adjustment of multiposition measurements using the Mahalanobis distance. Precision Engineering, 34 (1), 113-123.

[6] Zhang, F.M., Qu, X.H. (2012). Fusion estimation of point sets from multiple stations of spherical coordinate instruments utilizing uncertainty estimation based on monte carlo. Measurement Science Review, 12 (2), 40-45.

[7] D’Amato, R., Caja, J., Maresca, P., Gómez, E. (2014). Use of coordinate measuring machine to measure angles by geometric characterization of perpendicular planes. Estimating uncertainty. Measurement, 47, 598606.

[8] Shi, Y.Q., Sun, C.K., Wang, P., Wang, Z., Duan, H.X. (2011). High-speed measurement algorithm for the position of holes in a large plane. Optics and Lasers in Engineering, 50 (12), 1828-1835.

[9] Feng, M.C., Jin, Y., Zhai, C. (2011). Embedded realtime strain measurement system based on improved automated grid method. Chinese Journal of Scientific Instrument, 32 (12), 2874-2880.

[10] Janusek, D., Kania, M., Zaczek, R., Zavala-Fernandez, H., Zbiec, A., Opolski, G., Maniewski, R. (2011). Application of wavelet based denoising for T-wave alternans analysis in high resolution ECG maps. Measurement Science Review, 11 (6), 181-184.

[11] Liu, Z.G., Zhai, C., Hu, H.Z., Wang, J.P., Chu, J.R. (2011). Research on calibration method of LAMOST fiber robot. In Astronomical Adaptive Optics Systems and Applications IV, Proc. SPIE 8149. 
[12] Wang, M.X., Zhao, Y.H., Luo, A. (2012). LAMOST fiber unit positional precision passive detection exploiting the technique of template matching. In Modern Technologies in Space- and Ground-Based : Telescopes and Instrumentation II, Proc. SPIE 8450.

[13] Barone, S., Paoli, A., Razionale, A.V. (2013). Multiple alignments of range maps by active stereo imaging and global marker framing. Optics and Lasers in Engineering, 51 (2), 116-127.

[14] Guidi, G., Beraldin, J.A., Ciofi, S., Atzeni, C. (2003). Fusion of range camera and photogrammetry: A systematic procedure for improving 3-D models metric accuracy. IEEE Transactions on Systems, Man, and Cybernetics, Part B: Cybernetics, 33 (4), 667-676.

[15] Tao, C.V., Hu, Y. (2001). A comprehensive study of the rational function model for photogrammetric processing. Photogrammetric Engineering \& Remote Sensing, 67 (12), 1347-1357.

[16] Triggs, B., McLauchlan, P., Hartley, R., Fitzgibbon, A. (2000). Bundle adjustment-a modern synthesis. Vision Algorithms: Theory Practice, 1883, 298-372.

[17] Lourakis, M.I.A., Argyros, A.A. (2009). SBA: A software package for generic sparse bundle adjustment. ACM Transactions on Mathematical Software, 36 (1), 1-30.

[18] Luhmann, T. (2010). Close range photogrammetry for industrial applications. ISPRS Journal of Photogrammetry and Remote Sensing, 65 (6), 558569.

[19] Mouragnon, E., Lhuillier, M., Dhome, M., Dekeyser, F., Sayd, P. (2009). Generic and real-time structure from motion using local bundle adjustment. Image and Vision Computing, 27 (8), 1178-1193.

[20] Agarwal, S., Snavely, N., Seitz, S., Szeliski, R. (2010). Bundle adjustment in the large. In Computer Vision ECCV 2010, LNCS 6312, 29-42.

[21] Ji, Z., Boutin, M., Aliaga, D.G. (2006). Robust bundle adjustment for structure from motion. In IEEE International Conference on Image Processing. IEEE, 2185-2188.
[22] Marquardt, D. (1963). An algorithm for the leastsquares estimation of nonlinear parameters. SIAM Journal of Applied Mathematics, 11 (2), 431-441.

[23] Zhang, Y.Z. (2000). A flexible new technique for camera calibration. IEEE Transactions on Pattern Analysis and Machine Intelligence, 22 (11), 13301334.

[24] Tsai, M.J., Hung, C.C. (2005). Development of a highprecision surface metrology system using structured light projection. Measurement, 38 (3), 236-247.

[25] Tsai, R.Y. (1987). A versatile camera calibration technique for high-accuracy 3D machine vision metrology using off-the shelf TV cameras and lenses. IEEE Journal of Robotics and Automation, 3 (4), 323344.

[26] Huang, J.J., Wang, Z., Gao, Z.H., Gao, J.M. (2011). A novel color coding method for structured light 3D measurement. In Videometrics, Range Imaging, and Applications XI, Proc. SPIE 8085.

[27] Weng, J., Cohen, P., Henriou, M. (1992). Camera calibration with distortion models and accuracy evaluation. IEEE Transactions on Pattern Analysis and Machine Intelligence, 14 (10), 965-980.

[28] Hartley, R.I. (1997). In defense of the eight-point algorithm. IEEE Transactions on Pattern Analysis and Machine Intelligence, 19 (6), 580-593.

[29] Menudet, J.F., Becker, J.M., Fournel, T., Mennessier, C. (2008). Plane-based camera self-calibration by metric rectification of images. Image and Vision Computing, 26 (7), 913-934.

[30] Debei, S., Aboudan, A., Colombatti, G., Pertile, M. (2012). Lutetia surface reconstruction and uncertainty analysis. Planetary and Space Science, 71 (1), 64-72.

[31] Feng, M.C., Gu, Y.G., Zhai, C. (2013). Precise measurement of fibers position using bundle adjustment algorithm. In IEEE International Instrumentation and Measurement Technology Conference. IEEE, 576-580.

Received November 15, 2013. Accepted July 29, 2014. 